\title{
Neutron Radiography Studies of Water Self-Diffusion in Porous Medium
}

\author{
J.J. Milczarek, I. Fijae-Kirejczyk, M. Chojnowski \\ AND J. ŻOモA̧DEK \\ Institute of Atomic Energy, Świerk, 05-400 Otwock, Poland
}

Dedicated to Professor Jerzy Janik on the occasion of his 80th birthday

\begin{abstract}
The results of neutron radiography studies on macroscopic self-diffusion of water confined within rigid porous material reveal the substantial reduction in diffusion constant in comparison to the diffusion in large volume. The studies performed in the $30-50^{\circ} \mathrm{C}$ temperature range on fired clay brick of $30 \%$ porosity show decrease in diffusion constant to approximately $17 \%$ of its value for free space and the Archie exponent is 1.4. The Arrhenius law was confirmed and the activation energy was found of the same value as in the water bulk.
\end{abstract}

PACS numbers: 66.10.cg, 66.30.jj, 47.56.+r, 81.70.- $\mathrm{q}$

\section{Introduction}

The knowledge of self-diffusion parameters of water confined within a porous medium is important for chemical engineering and optimizing of soil moisture as well as basic science [1-4]. The slowing down of the diffusion advancing in a liquid filling up the voids within solid matrix of porous medium in comparison to the process proceeding in a large volume is known very well. The experimental data on the diffusion parameters are of a crucial importance for any theory of the diffusion in liquid in confined geometries [3-7]. Most of the accurate measurements of the diffusion coefficient were obtained with application of radioactive isotopic tracer [8], specialized NMR techniques as pulsed-field-gradient spin-echo $[9,10]$ or neutron quasielastic scattering [11]. The NMR methods provide the data on diffusion on small distances $(\sim 10 \mu \mathrm{m})$ and short times $(\sim 100 \mu \mathrm{s})$ [9]. In this work we report for the first time on the neutron radiography measurements of selfdiffusion of water in porous medium observed on macroscopic distances $(\sim 1 \mathrm{~cm})$.

The main aim of our work was to apply the dynamic neutron radiography in quantitative studies of water self-diffusion in a porous system. The application 
of the neutron radiography employs the marked optical contrast between ordinary and heavy water for thermal neutrons. The contrast comes mainly from large incoherent scattering of thermal neutrons by systems containing hydrogen nuclei, the effect almost absent in case of deuterons.

Our first observations of the water self-diffusion carried out with the neutron radiography yielded only rough estimate of the self-diffusion coefficient at room temperature [12]. In the present work the system studied was held in stabilized conditions and the measurements were performed at $30,35,40,45$, and $50^{\circ} \mathrm{C}$. One should bear in mind that the diffusion is rather slow phenomenon in comparison to the spontaneous imbibition driven by capillary force. The times of observations are then almost one hundred times longer than that needed in case of the capillary forces induced migration of liquid in the same porous system.

\section{Experimental}

The studies were performed on a sample of fired clay brick of effective porosity $\sim 30 \%$ ( $36 \%$ as determined by destructive mercury intrusion), of the same kind as used previously as the medium in spontaneous imbibition studies [12-14]. The effective capillary radius of the sample as determined from the rate of water imbibition was $\sim 5.5 \mathrm{~nm}$. The pore boundary surface has a fractal structure with the fractal dimension $D_{\mathrm{f}} \sim 2.4$ estimated with the small angle neutron scattering and analysis of the spatial distribution of water during imbibition [15].

(a)

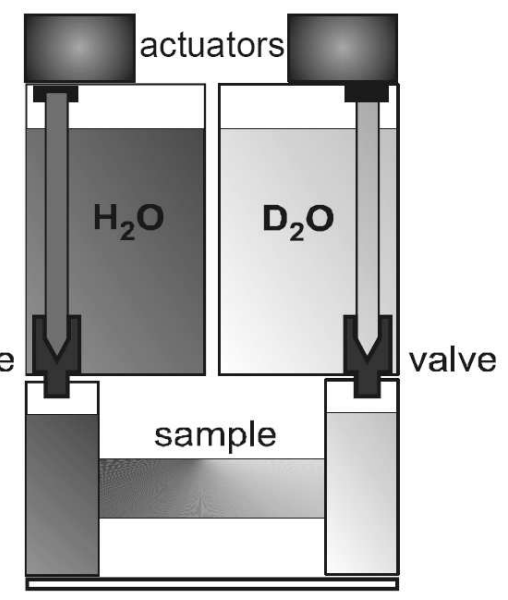

(b)

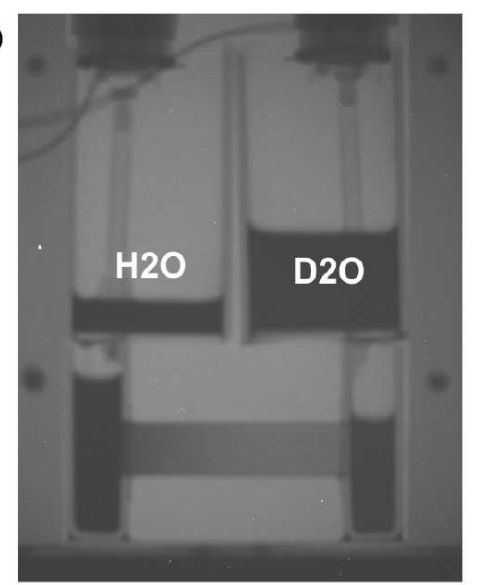

Fig. 1. The sketch of the experimental setup (a), and the neutron radiogram taken after $71 \mathrm{~h}$ of the experiment at $50^{\circ} \mathrm{C}(\mathrm{b})$. Due to water evaporation the upper containers are only partially filled with liquids after $70 \mathrm{~h}$.

The experimental setup comprised the parallelepiped $54 \times 12 \times 12 \mathrm{~mm}$ sample of the porous material with two containers fixed to its ends (Fig. 1). The sample was initially dried for approximately $70 \mathrm{~h}$ at $80^{\circ} \mathrm{C}$. Then it was saturated for 
a

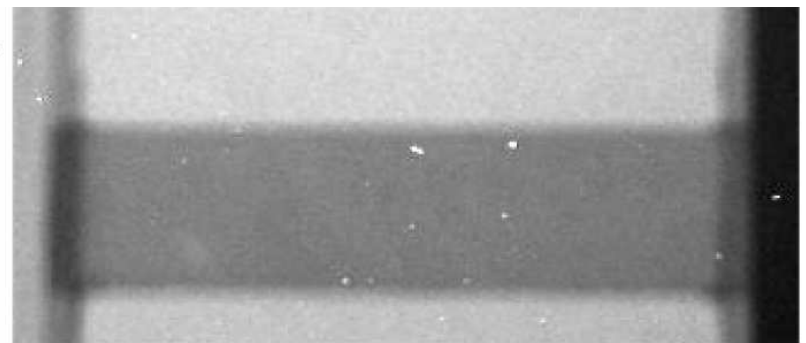

b

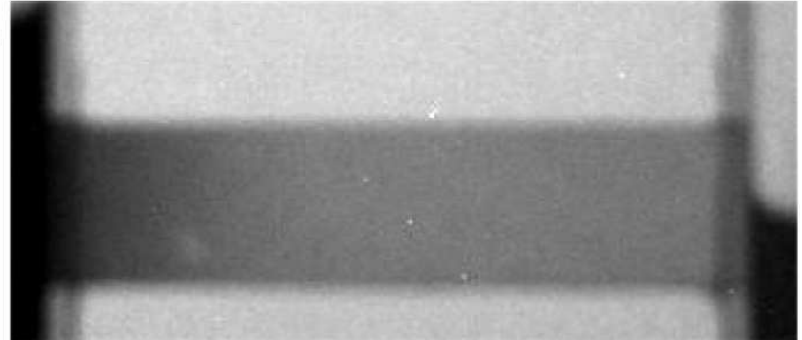

c

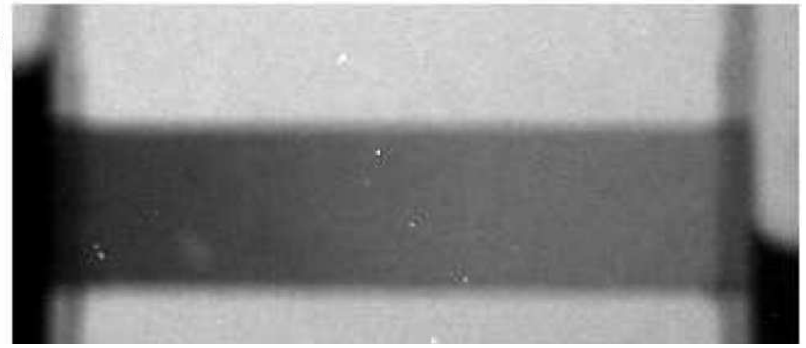

Fig. 2. The radiograms of the sample at $50^{\circ} \mathrm{C}$ taken before (a), and at two different times 23 (b), and $71 \mathrm{~h}$ (c), after flooding the left container with $\mathrm{H}_{2} \mathrm{O}$.

$24 \mathrm{~h}$ with $\mathrm{D}_{2} \mathrm{O}$ by filling both containers with heavy water. Before starting the experiment the two upper containers with valves enabling to fill and supplement the sample containers with appropriate liquid were attached to the system. The whole setup was placed inside the special thermal cell and kept in a stabilized temperature $\left(30,35,40,45\right.$, and $\left.50^{\circ} \mathrm{C}\right)$ for $3 \mathrm{~h}$ and then put in contact with $\mathrm{H}_{2} \mathrm{O}$ at one of its ends. In order to avoid evaporation of $\mathrm{D}_{2} \mathrm{O}$ from the sample its sides were covered with acrylic paint and the second end of the specimen was in permanent contact with $\mathrm{D}_{2} \mathrm{O}$ filled container (Figs. 1 and 2).

The presence of the $\mathrm{H}_{2} \mathrm{O}$ in the material can be observed due to the large optical neutron contrast between $\mathrm{H}_{2} \mathrm{O}$ and $\mathrm{D}_{2} \mathrm{O}$. The neutron radiograms were recorded for over $80 \mathrm{~h}$ with the dynamical neutron radiography station at MARIA reactor $[12,13]$. In first few hours after filling the $\mathrm{H}_{2} \mathrm{O}$ container with the liquid we observed in the radiograms a dark region that emerged at the $\mathrm{H}_{2} \mathrm{O}$ immersed end and progressed slowly towards the sample's end in contact with $\mathrm{D}_{2} \mathrm{O}$ (Fig. 2).

The brightness of the pixels on the axial line of the sample image was used as a measure of the $\mathrm{H}_{2} \mathrm{O}$ volumetric content in a sample. The dependence of the 
volumetric content of ordinary water $\theta(x, t)$ at some time $t$ on the position $x$ along the axis was determined according to the standard procedure $[12,13]$ :

$$
\theta(x, t)=\frac{1}{W\left(\Sigma_{\mathrm{H}}-\Sigma_{\mathrm{D}}\right)} \ln \frac{I_{\mathrm{D}}(x)}{I_{\mathrm{H}}(x, t)},
$$

with $I_{\mathrm{D}}(x)$ and $I_{\mathrm{H}}(x, t)$ denoting the intensity of the neutrons leaving the sample saturated with $\mathrm{D}_{2} \mathrm{O}$ and containing $\mathrm{H}_{2} \mathrm{O}$ molecules at the position after some time $t$, respectively. The neutron intensities, $I_{\mathrm{D}}, I_{\mathrm{H}}$ were determined as the differences
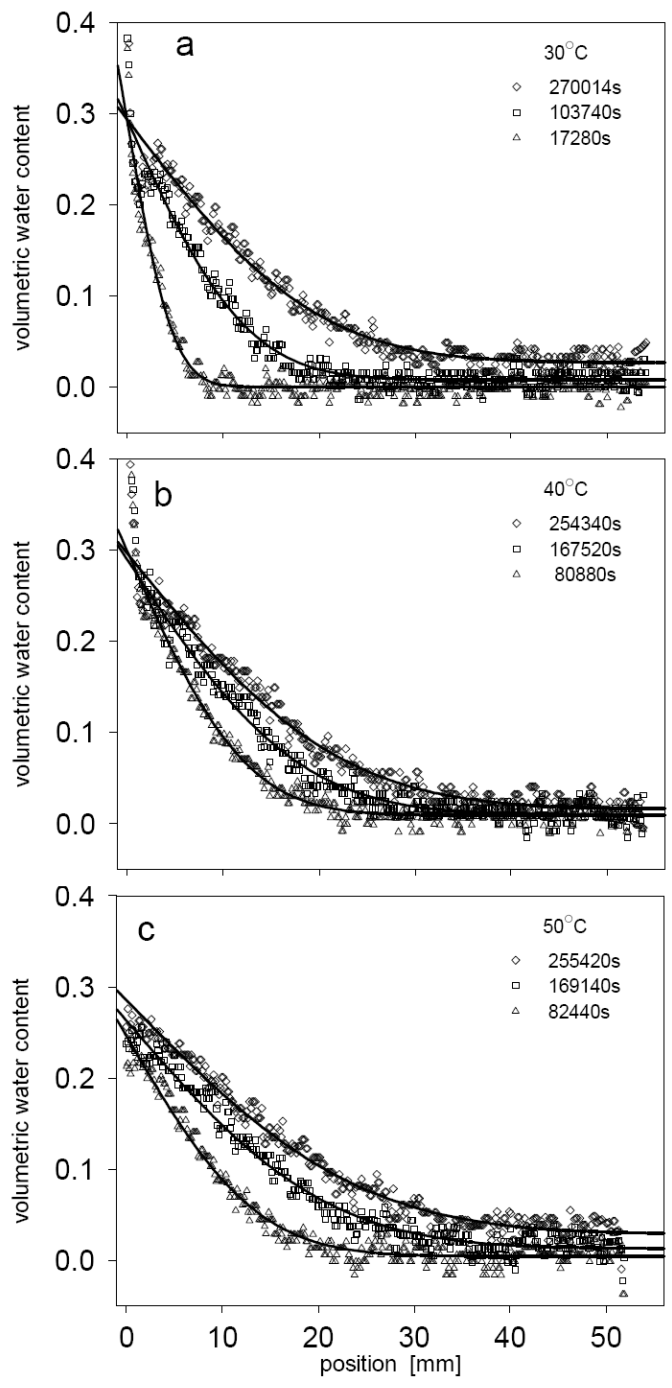

Fig. 3. The experimental spatial distributions of $\mathrm{H}_{2} \mathrm{O}$ content along sample axis (points) at $30^{\circ} \mathrm{C}(\mathrm{a}), 40^{\circ} \mathrm{C}(\mathrm{b})$, and $50^{\circ} \mathrm{C}$ (c), fitted with the solution of the diffusion equation (solid lines). 
between pixel brightness of corresponding image and the average brightness of the radiogram taken with shielded neutron beam. $W, \Sigma_{\mathrm{H}}$, and $\Sigma_{\mathrm{D}}$ are sample width and macroscopic scattering length of $\mathrm{H}_{2} \mathrm{O}$ for neutrons, respectively. The images were calibrated and the average distance between neighboring pixels corresponds to $0.153 \mathrm{~mm}$.

The experimental distributions of $\mathrm{H}_{2} \mathrm{O}$ were determined for at least five different times (ranging from 2 to $75 \mathrm{~h}$ ) after beginning of the process for each of the five experimental temperatures. The data (Fig. 3) were analyzed in terms of the simple solution

$$
\theta(x, t)=\theta_{0} \operatorname{erfc}\left(\frac{x}{2 \sqrt{D t}}\right)+\theta_{\mathrm{b}}
$$

of the diffusion equation, with the diffusion coefficient $D$, initial water saturation $\theta_{0}$ and saturation background $\theta_{\mathrm{b}}$ as the fitting parameters. The solution corresponds to the boundary condition $\theta(x=0, t)=$ const and $\theta(x=\infty, t)=\theta_{\mathrm{b}}$.

The irregularities observed in the experimental curves are the main reason for rather large error bars for estimated quantities. They are due to the medium value of the neutron flux density at the sample $\left(5 \times 10^{6} \mathrm{~cm}^{-2} \mathrm{~s}^{-1}\right)$ and the 8-bit

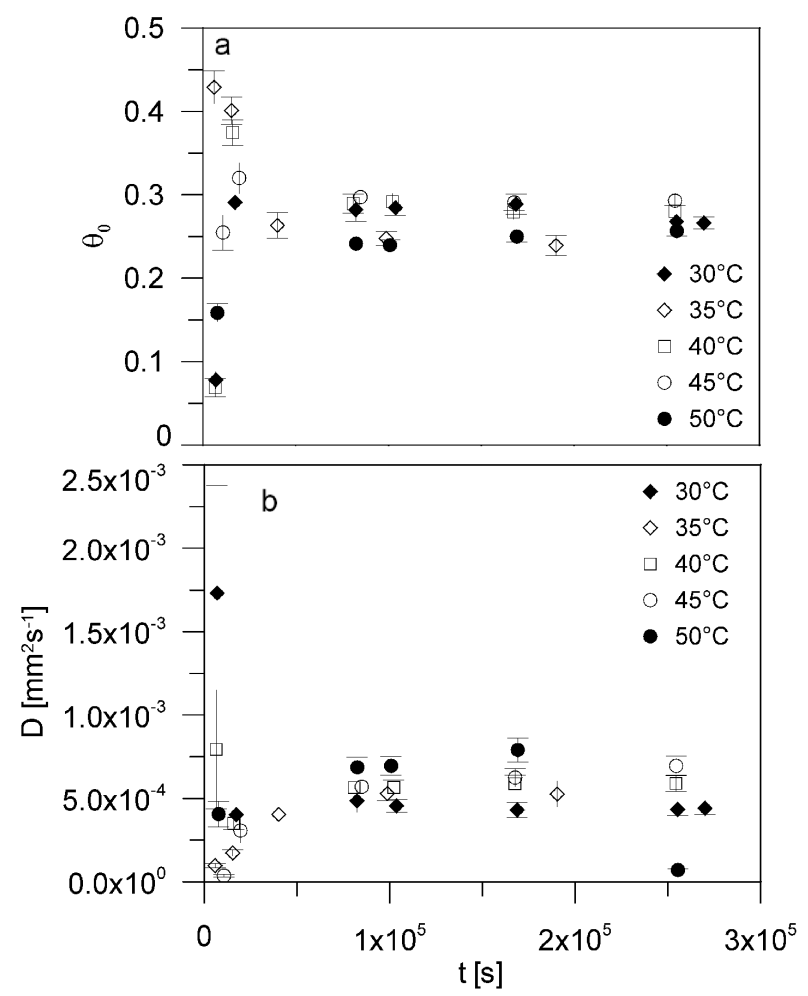

Fig. 4. The dependence of the fit parameters: $x=0$ boundary water content $\theta_{0}(\mathrm{a})$, and diffusion coefficient $D(\mathrm{~b})$, on time $t$ elapsed since the beginning of the process. 
coding of the gray scale used in the camera software. The deficiency could be overcome with application of the higher neutron flux and more dynamic (12-bit) encoding.

Due to the presence of the neutrons scattered on the $\mathrm{H}_{2} \mathrm{O}$ container and sparse data in the narrow region of $5 \mathrm{~mm}$ width near $x=0$ the determined parameters $D$ and $\theta_{0}$ vary substantially with the time for the initial stages of the process (Fig. 4) at any temperature considered. One should note that for longer times these values stabilize and $\theta_{0}$ is close to the 0.3 , the effective porosity of the sample. The background $\theta_{\mathrm{b}}$ increases with the time of the process. For further analysis we have assumed that at each temperature the macroscopic diffusion of the $\mathrm{H}_{2} \mathrm{O}$ in the porous medium saturated with $\mathrm{D}_{2} \mathrm{O}$ is described at each temperature by the average values of the diffusion coefficient determined for the longest times. This assumption yields the values of the diffusion constant within $12 \%$ error bars.

\section{Discussion}

We have assumed that the determined diffusion constant is the water selfdiffusion constant. Since the error bars for our values are of approximately $12 \%$ and the correction factor accounting for the difference in mass between $\mathrm{H}_{2} \mathrm{O}$ and $\mathrm{D}_{2} \mathrm{O}$ is approximately 0.95 , this assumption is reasonable for the discussion of the behavior of the diffusion coefficient.

First of all, we have found that the diffusion coefficient determined for $\mathrm{H}_{2} \mathrm{O}$ diffusing in $\mathrm{D}_{2} \mathrm{O}$ filling the pores of the porous medium is substantially smaller than the values of the self-diffusion coefficients in water given for large volumes. Specifically, the determined water self-diffusion coefficient in fired brick at $30^{\circ} \mathrm{C}$ is equal to $4.2 \times 10^{-4} \mathrm{~mm}^{2} \mathrm{~s}^{-1}$ and is approximately 6 times smaller than that for the free space $\left(2.6-2.9 \times 10^{-3} \mathrm{~mm}^{2} \mathrm{~s}^{-1}\right)[8,16]$. The reduction of transport coefficients (like electrical conductivity), for materials confined within pore boundaries in porous media is well known effect and is conventionally discussed in terms of the Archie law $[9,17,18]$. Written in terms of the diffusion coefficient the Archie law links the diffusion constant for porous system with the system porosity

$$
\frac{D_{\text {porous }}}{D_{\text {bulk }}}=A \Phi^{m}
$$

where $D_{\text {bulk }}$ is the diffusion constant measured in large volume. The Archie law exponent $m$ is equal to 1.4 in our case, a value close to that found for electrical conductivity of brine filling sedimentary rocks of different porosity [18]. However, the conventional description of reduction of transport coefficients by only one variable, porosity, should be considered with many qualifications [9]. In the case of water self-diffusion the mean length of a diffusive jump in the direction of diffusion is approximately $0.4 \mathrm{~nm}$ [16] whereas the radius of pores determined within capillary migration model for our medium is $5.5 \mathrm{~nm}$ and should not restrict the thermal motion of water molecules substantially. One should expect that the tortuosity i.e. the ratio of the length of real average molecule path to the 
shortest distance measured along the direction of the molecule's effective motion is much more important. The problem is that the estimation of the tortuosity from the independent transport coefficients measurements is not free from model assumptions [17].

Ignoring inaccuracies observed for short times of the process no deviations from the normal diffusion were noticed. The fractal nature of the pore surface boundaries did not produce the anomalous diffusion effects at least for the longer times of observations.

We have found that the temperature dependence of the water self-diffusion coefficient in the fired clay brick can be fitted with Arrhenius law (Fig. 5):

$$
D=D_{0} \exp \left(-\Delta E_{0} / k_{\mathrm{B}} T\right)
$$

yielding the activation energy $\Delta E_{0}$ of $5 \pm 0.8 \mathrm{kcal} \mathrm{mol}^{-1}$, and $D_{0}$ of $1.9 \pm$ $0.6 \mathrm{~mm}^{2} \mathrm{~s}^{-1}$. The estimated value of activation energy is approximately $10 \%$ higher than that $\left(4.6 \mathrm{kcal} \mathrm{mol}^{-1}\right)$ reported for the free space diffusion $[8,16]$.

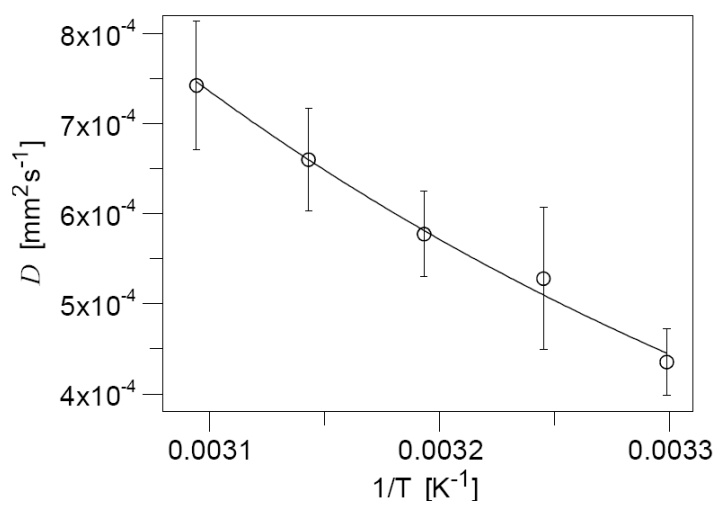

Fig. 5. The self-diffusion coefficient of water in the fired clay brick plotted against inverse temperature. The solid line is the Arrhenius law fit.

For some applications it might be important that within the investigated range of the temperature, the temperature dependence of the determined water self-diffusion constant for porous medium can be also described by the linear function

$$
D(T)\left[\mathrm{mm}^{2} \mathrm{~s}^{-1}\right]=(1.5 \pm 0.1) \times 10^{-5} T\left[{ }^{\circ} \mathrm{C}\right]-(8.4 \pm 0.3) \times 10^{-6} .
$$

The activation energy determined from the linear approximation is consistent with power expansion of the Arrhenius law

$$
D(T)=D\left(T_{0}\right)\left[1+\frac{\Delta E_{0}}{k_{\mathrm{B}} T_{0}^{2}}\left(T-T_{0}\right)\right],
$$

which yields $\Delta E_{0}$ equal to $4.9 \pm 0.8 \mathrm{kcal} \mathrm{mol}^{-1}$ if one takes $T_{0}=313 \mathrm{~K}$. 


\section{Summary}

We determined the self-diffusion constant for water confined within porous medium. The self-diffusion in the porous material is substantially slower than that occurring in free space and can be described by the Archie exponent equal to 1.4. Despite fractal nature of the pore boundaries no features of anomalous diffusion were observed. The temperature dependence of the diffusion coefficient conforms to the Arrhenius law and the activation energy is close to that given for free space diffusion as should be expected from the thermal motion nature of the diffusion. The hints for improving the accuracy of determination of the diffusion parameters with the neutron radiography have been given.

\section{References}

[1] G.A. Aksielrud, M.A. Altszuler, Mass Transfer in Porous Media, WNT, Warsaw 1987 (in Polish).

[2] D. Kirkham, W.L. Powers, Advanced Soil Physics, Wiley-Interscience, New York 1972.

[3] R.E. Cunningham, R.J.J. Williams, Diffusion in Gases and Porous Media, Plenum Press, New York 1980.

[4] P.N. Sen, in: Porous Media in Processes with Long- Range Correlations. Theory and Applications, Eds. G. Rangarajan, M. Ding, Springer-Verlag, Berlin 2003, p. 181.

[5] P. Liu, E. Harder, B.J. Berne, J. Phys. Chem. B 108, 6595 (2004).

[6] R. Zangi, J. Phys., Condens. Matter 16, S5371 (2004).

[7] M. Vergeles, G. Szamel, J. Chem. Phys. 110, 3009 (1999).

[8] R. Pruppacher, J. Chem. Phys. 56, 101 (1972).

[9] F. D'Orazio, S. Bhattacharja, W. P. Halperin, R. Gerhardt, Phys. Rev. Lett. 63, 43 (1989); Phys. Rev. B 42, 6503 (1990).

[10] P.N. Sen, L.M. Schwartz, P.P. Mitra, B.I. Halperin, Phys. Rev. B 49, 215 (1994).

[11] M. Bée, Quasielastic Neutron Scattering, Adam Hilger, Bristol 1988.

[12] J.J. Milczarek, A. Czachor, A. El-Ghany El Abd, Z. Wiśniewski, Nucl. Instrum. Methods Phys. Res. A 542, 232 (2005).

[13] A. El-Ghany El Abd, J.J. Milczarek, J. Phys. D: Appl. Phys. 37, 2305 (2004).

[14] J. Ridgway, P.A.C. Gane, A. El-Ghany El Abd, A. Czachor, Transport Porous Media 63, 503 (2006).

[15] J.J. Milczarek, A. El-Ghany El Abd, in: Neutron Scattering and Complementary Methods in Investigations of Condensed Phase, Vol. 1, Ed. J. Chruściel, University of Podlasie Publ. House, Siedlce 2003, p. 175.

[16] D. Eisenberg, W. Kauzmann, The Structure and Properties of Water, Clarendon Press, Oxford 1969.

[17] F.A.L. Dullien, Porous Media. Fluid Transport and Pore Structure, Academic Press, New York 1979.

[18] H. Thompson, A.J. Katz, C.E. Krohn, Adv. Phys. 36, 625 (1987). 